\title{
A multiplexed quantum repeater based on absorptive quantum memories
}

\author{
Zhengwei ZHOU \\ Department of Optics and Optical Engineering, University of Science and Technology of China, Hefei 230026, China
}

Received 17 June 2021/Accepted 23 June 2021/Published online 29 June 2021

Citation Zhou Z W. A multiplexed quantum repeater based on absorptive quantum memories. Sci China Inf Sci, 2021, 64(11): 217501, https://doi.org/10.1007/s11432-021-3287-6

Quantum entanglement is the fundamental resource of quantum communication. Due to the inevitable channel loss, the current entanglement distribution distance based on optical fiber channel is limited to the order of $100 \mathrm{~km}$, which hinders the construction of large-scale and global quantum networks. To overcome this difficulty, scientists have proposed the strategy of so-called quantum repeater [1], where the long-distance entanglement distribution is divided into multiple short-distance basic links. In the quantum repeater, the entanglement between the two end points of the basic links is established firstly, and then the neighboring elementary links are connected through entanglement swapping to establish the long-distance entanglement step by step, finally, the high-quality and long-distance entangled states are obtained by virtue of the entanglement purification process. Previous demonstrations of the elementary link of a quantum repeater are based on emissive quantum memories, that is to say, the entangled light source is integrated with quantum memory which is usually the cold atomic ensemble or a single-atom system. The photons are emitted from the memory and their quantum states are entangled. If a single-atom system is employed as the memory, it is difficult to achieve multiplexed operations; if an atom ensemble system is used, the light-emitting process is probabilistic with low efficiency.

In order to overcome this difficulty, the recent work of the research groups of $\mathrm{Li}$ and Zhou [2] from the University of Science and Technology of China constructed the fundamental link of quantum repeater based on absorptive quantum memory for the first time. Such kind of quantum memory is flexible with the choice of entanglement sources while retaining the capability of multiplexed operations, and therefore is more efficient for quantum repeater applications. In their experiment, two sets of entangled photon pairs were prepared based on the spontaneous parametric down-conversion. One photon of each photon pair is sent to the corresponding solid-state quantum memory for stor- age, and the other is sent to the intermediate site for Bell state measurement (BSM). A click of the BSM heralds the successful distribution of entanglement between two solidstate quantum memories through entanglement swapping. In order to demonstrate the technical advantages of such kind of architecture, they introduced 4 multiplexed temporal modes in the experiment, which increased the rate of entanglement distribution by 4 times. The fidelity of the entangled state between the two solid-state quantum memories reaches $80.4 \%$.

This experiment verifies the possibility of using the absorptive quantum memories to construct quantum repeater, and directly demonstrates the advantage of enhancement of communication rate using a multiplexed quantum repeater. However, in the current experiment, they used a probabilistic entanglement light source, which imposed a limit on the rate of entanglement distribution. Now they are working on a deterministic entangled light source based on semiconductor quantum dots to realize the multiplexed quantum storage of deterministic entangled photons. This will be a great attempt full of a beautiful vision. On the one hand, the use of deterministic entanglement light sources can significantly increase the entanglement distribution rate of a quantum repeater. On the other hand, the combination of quantum dots and rare earth ion-doped crystals can fully demonstrate the advantages of all solid-state systems, including system stability, easy processing, and easy integration, thereby improving the stability of the quantum repeater system. Eventually, it is expected to build an all-solid-state high-speed quantum repeater network.

\section{References}

1 Briegel H J, Dür W, Cirac J I, et al. Quantum repeaters: the role of imperfect local operations in quantum communication. Phys Rev Lett, 1998, 81: 5932-5935

2 Liu X, Hu J, Li Z F, et al. Heralded entanglement distribution between two absorptive quantum memories. Nature, 2021, 594: 41-45 